\title{
Maternal Influenza Infection During Pregnancy Impacts Postnatal Brain Development in the Rhesus Monkey
}

\author{
Sarah J. Short, Gabriele R. Lubach, Alexander I. Karasin, Christopher W. Olsen, Martin \\ Styner, Rebecca C. Knickmeyer, John H. Gilmore, and Christopher L. Coe \\ Department of Psychology (SJS, GRL, CLC), Harlow Center for Biological Psychology; \\ Department of Pathobiological Sciences (AIK, CWO), School Veterinary Medicine, University of \\ Wisconsin, Madison, Wisconsin; and the Department of Psychiatry (MS, RCK, JHG), \\ Neurosciences Hospital, University of North Carolina, Chapel Hill, North Carolina
}

\begin{abstract}
Background-Maternal infection with influenza and other pathogens during pregnancy has been associated with increased risk for schizophrenia and neurodevelopmental disorders. In rodent studies, maternal inflammatory responses to influenza affect fetal brain development. However, to verify the relevance of these findings to humans, research is needed in a primate species with more advanced prenatal corticogenesis.
\end{abstract}

Methods-Twelve pregnant rhesus monkeys were infected with influenza, A/Sydney/5/97 (H3N2), 1 month before term (early third trimester) and compared with 7 control pregnancies. Nasal swabs and blood samples confirmed viral shedding and immune activation. Structural magnetic resonance imaging was conducted at 1 year; behavioral development and cortisol reactivity were also assessed.

Results-Maternal infections were mild and self-limiting. At birth, maternally derived influenzaspecific immunoglobulin $\mathrm{G}$ was present in the neonate, but there was no evidence of direct viral exposure. Birth weight and gestation length were not affected, nor were infant neuromotor, behavioral, and endocrine responses. However, magnetic resonance imaging analyses revealed significant reductions in cortical gray matter in flu-exposed animals. Regional analyses indicated the largest gray matter reductions occurred bilaterally in cingulate and parietal areas; white matter was also reduced significantly in the parietal lobe.

Conclusions-Influenza infection during pregnancy affects neural development in the monkey, reducing gray matter throughout most of the cortex and decreasing white matter in parietal cortex. These brain alterations are likely to be permanent, given that they were still present at the monkeyequivalent of older childhood and thus might increase the likelihood of later behavioral pathology.

\section{Keywords}

Brain development; fetal; influenza; pregnancy; schizophrenia

Physical and psychological challenges to maternal wellbeing during pregnancy can adversely affect fetal brain development, increasing the risk for behavioral and psychiatric disorders. In addition to the known effects of many drugs and environmental toxicants, viral

\footnotetext{
(C) 2010 Society of Biological Psychiatry

Address correspondence to Sarah J. Short, Ph.D., University of North Carolina at Chapel Hill, 7023 Neurosciences Hospital, 101 Manning Drive, Campus Box 7160, Chapel Hill, NC 27599-7160; sjshort@med.unc.edu..

The authors report no biomedical financial interests or potential conflicts of interest.

Supplementary material cited in this article is available online.
} 
and bacterial infections are among the natural factors thought to pose some risk to the expectant mother and developing baby. Mounting evidence from both animal and human studies has specifically implicated influenza infection as one viral pathogen of potential concern on the basis of increased risk for neuropsychiatric diseases such as schizophrenia (1-6). It has been estimated that approximately $11 \%$ of pregnant women are infected with influenza at some point during gestation (7). However, the full consequences for brain and behavioral development have been difficult to ascertain empirically due to variation in strain virulence, differential effects induced by gestational timing of infection, and the long delay until first clinical diagnosis in the offspring. One case/control study of adults with schizophrenia born between 1959 and 1966 used banked serum samples to detect the presence of maternal antibody against influenza during pregnancy and concluded that infection posed a four-fold to seven-fold increase in risk (8). This significant effect was found primarily during the first half of pregnancy, but reports on other cohorts born during different years, especially after the major pandemic in 1957, suggest that the risks from infection might be as pronounced in the second and third trimesters $(5,9,10)$.

Concerns from human studies have been corroborated by infection experiments in rodents, which demonstrated that off-spring from influenza-exposed pregnancies manifest many neural abnormalities (11), including decreased cortical thickness and smaller hippocampal volumes $(12,13)$. Microarray analyses of brains from exposed mice indicate that the decreased corticogenesis is associated with both up- and downregulation of genes for structural proteins, including reelin and others important for neuronal migration, synaptogenesis, and gliogenesis $(14,15)$. In addition, monoamine neurotransmitter levels are altered, including decreased serotonin in the hippocampus, nucleus accumbens, and lateral globus pallidus as well as increased dopamine in prefrontal cortex and lateral globus pallidus (16). Behavioral abnormalities have been found in tests of emotionality and sensory-motor integration, including poorer adaptation to acoustical startle in prepulse inhibition paradigms $(6,17)$. However, directly extrapolating these results to humans is difficult because rodent pups are quite immature at birth, especially with regard to brain development (18). When compared with a human fetus in the third trimester, the equivalent maturational point actually occurs postnatally (19). Thus, it is critical to investigate the neural impact of maternal infection in a primate that has greater cortical maturation during the prenatal period. Many infection-induced effects on the developing cortex in humans are likely due to a derailing of the extensive pruning and apoptosis that takes place after neurons are initially overproduced in the fetal brain and to a curtailing of the ongoing dendritogenesis and synaptogenesis (20).

Influenza is a zoonotic virus capable of infecting many species, including birds and farm animals, but it is important to appreciate that the nature of the infection and symptom severity varies markedly. In mice, ferrets, and pigs, influenza infection early in pregnancy often results in miscarriage or in some fetal loss, typically evident by a reduced litter size $(21,22)$. Virulent strains of influenza can sometimes have this lethal effect in humans as well, especially if there is a secondary bacterial pneumonia (23), although the primary concern for most pregnancies would be the impact on viable offspring. Recent reports of abnormal brain development in mouse models have mostly used a neurovirulent strain of influenza, A/NWS/33 (H1N1), which results in a potent infection $(12,14)$. Thus, in addition to employing a monkey model, it was important to determine whether similar neural effects are evident after maternal infections with the less pathogenic influenza strains that have circulated among humans in recent years. Beyond these issues of viral strain and comparability of fetal maturation, the similarity of placental functioning in the monkey is of importance. Because influenza infections are restricted largely to the airways, most investigators believe that the adverse effects on fetal development are mediated either by disruptions of placental physiology or increased cytokine activity in the fetal compartment. 
Only a few influenza strains have ever been found to transfer across the placenta and possess the neurotropism needed to directly infect fetal neural tissues $(24,25)$. However, concerns have been raised about the transfer of maternal antibody into the fetal compartment, which was monitored in this study.

The rhesus monkey meets the criteria listed in the preceding text: 1) it was possible to infect the female monkeys with a human-derived H3N2 strain; 2) similar to humans, this species has a hemochorial placenta with greater penetrance and vascular permeability, enabling intimate communication between maternal and fetal physiology (26-30); and 3) it gives birth to one infant at a time with advanced brain maturation $(18,31,32)$. Our focus was on the latter half of pregnancy when the maternal inflammatory response in gravid women is actually larger, as evinced by greater release of tumor necrosis factor after stimulating their leukocytes with endotoxin (33). Our primary goal was to determine whether infection at this point in pregnancy would result in persistent brain alterations large enough to be visualized via neuroimaging.

\section{Methods and Materials}

\section{Subjects and Housing}

Nineteen mother and infant pairs of rhesus monkeys (Macaca mulatta) at the Harlow Primate Laboratory were evaluated. Healthy, multiparous adult females (5-15 years old) were time-mated with single sires to generate infants: 12 experimental ( $n=7$ males) and 7 control $(n=3$ males). Pregnant dams remained undisturbed with the exception of a 2-week quarantine period during the infection phase. Control mothers received the same handling procedures but received saline intranasally instead of virus.

\section{Virus Generation and Viral Infection}

Embryonated chicken eggs were inoculated with stock virus (A/Sydney/5/97 (H3N2)) in phosphate-buffered saline with antibiotics. Virus generation was confirmed by hemagglutinin assay and viral titer quantified in Egg Infectious Dose units $\left(\mathrm{EID}_{50}\right)$. During week 17 of their 24-week pregnancy, gravid females were immobilized briefly with ketamine hydrochloride (10 mg/kg intramuscular [IM]) and exposed intranasally to either 1 $\times 10^{7} \mathrm{EID}_{50}$ via $1-\mathrm{mL}$ infusion of a virus-containing phosphate-buffered saline solution or to $1 \mathrm{~mL}$ saline (sham infection).

\section{Confirmation of Viral Infection}

Maternal infection was verified with three measures: presence of virus in nasal secretions and serum levels of neopterin and virus-specific antibody. Specifically, the virus in nasal secretions was quantified on Days 3, 7, and 14. A sterile Dacron swab was inserted into each nostril and then into transport media. Viral titer was determined by inoculating embryonated eggs $\left(\mathrm{EID}_{50} / \mathrm{mL}\right)$. Systemic inflammatory activity was assessed by measuring neopterin on Days 3 and 7 with an enzyme-linked immunosorbent assay (American Lab Products, Salem, New Hampshire). Antibody increases on Day 14 and at term further confirmed immune activation specific to influenza. Virus-specific immunoglobulin $\mathrm{G}(\mathrm{IgG})$ and immunoglobulin $\mathrm{M}$ (IgM) were determined by enzyme-linked immunosorbent assay (Diagnostic Automation, Calabasas, California). Briefly, sera were diluted 1:100 and $100 \mu \mathrm{L}$ pipetted into microtiter plates coated with immobilized A/Sydney antigen. After 1-hour incubation, anti-human-IgG or IgM peroxidase conjugate was added, and bound antibody reacted with tetramethylbenzidine. Color was quantified spectrophotometrically at 450-nm wavelength (Dynatech, Chantilly, Virginia). Optical densities were expressed in arbitrary units $(\mathrm{U} / \mathrm{mL})$ relative to control subjects. Presence of IgM would indicate placental transfer of virus and a primary immune response by the fetus. 


\section{Infant Measures}

Neonatal Behavior and Neuromotor Assessment-Behavioral maturation, attentional processes, and neuromotor reflexes were determined at 2 weeks of age with a standardized test battery. Infants were scored on 18 elements grouped into four categories: Orientation, Motor Maturity, Motor Activity, and Temperament (34). Previous studies have shown this test detects the effects of prenatal exposure to maternal stress or alcohol (35).

Mother-Infant Observations-Infants were observed (1-4 months) with their mothers in their home cages for three 5-min periods/week, scoring interactions on a laptop computer. Important measures reflective of the development of independence and infant emotionality included time spent on the mother, maternal retrieval and rejection, infant vocalizations, and initiation of environmental exploration (36). See Table S1 in Supplement 1 for Behavioral Ethogram.

Cortisol Measurements-Infants were weaned from the mother by 7 months and then housed in social groups of 6-8 juveniles (equivalent to mid-childhood in humans). At approximately 18 months, six blood samples $(<1 \mathrm{~mL})$ were collected across a 1-week period to determine whether adrenal activity differed. Samples were collected within 2 min and included: Day 1 Basal—at 3:00 pm in their home cage; Day 3 Acute Stress—at 4:00 pm, 1 hour after relocating a pair to an unfamiliar room; Day 5 Acclimation—at 8:00 AM and 3:00 PM to assess diurnal rhythm and adjustment to novel housing conditions; Day 7 Dexamethasone Suppression Test (DST) - at 8:00 AM and 3:00 PM on the day after dexamethasone sodium phosphate $(.25 \mathrm{mg} / \mathrm{kg}$, IM at 2000 (Day 6). Cortisol was determined in duplicate via iodinated radioimmunoassay (DiaSorin, Stillwater, Minnesota).

\section{Brain Scan Procedures and Magnetic Resonance Methods}

At approximately 1 year of age, (equivalent to 5-7 years in humans), magnetic resonance imaging (MRI) scans were conducted at the Waisman Laboratory for Brain Imaging and Behavior. Imaging was performed on a GE Signa 3-T scanner (Milwaukee, Wisconsin) with high-resolution coronal three-dimensional, Inversion-Recovery-Prepped, fast spoiled gradient recalled sequences for T1- and T2-weighted scans across the entire cranium. See Supplement 1 for imaging parameters. Scans were aligned with a three-plane localizer. Monkeys were immobilized with ketamine (10 mg/kg IM) and medatomidine (50 $\mu \mathrm{g} / \mathrm{kg} \mathrm{IM})$ for the 1-hour scan. A stereotaxic platform within an 18-cm diameter quadrature extremity coil ensured identical orientation. Minimal head tilt or yaw was verified by visualizing sagittal and coronal images.

\section{Image Processing and Analysis}

An unbiased structural atlas created specifically for juvenile rhesus brains served as the standardized template for determining probabilistic tissue maps, lobar parcellations, and subcortical structures (for atlas construction see Styner et al. [37]). This information was then applied to each subject's structural image via affine, followed by deformable registration. The affinely transformed probabilistic tissue maps were employed for a joint T1- and T2-based tissue segmentation of gray matter (GM), white matter (WM), and cerebrospinal fluid. Parcelled lobar and sub-cortical structure segmentations were calculated via the deformable registration to determine whether brain effects were global or regionally selective (Figure 1). Volumetric measurements of tissue, lobar regions, and subcortical structures were extracted automatically from the segmentations. Briefly, the goals were: 1) quantification of overall brain size and cortical GM and WM, 2) quantifying GM and WM within parcelled regions, and 3) determination of subcortical structures and lateral ventricle volumes. The automatic methods were validated against expert segmentations (38). 


\section{Statistical Analysis}

Group differences were determined with between and repeated measures of analyses of variance, corrected with Green-house-Geisser when appropriate. Prenatal Condition and Infant Sex were included as between factors with the exception of mother-infant behavior where the absence of gender differences precluded the need to consider gender. Analyses of MRI data included cortical regions (prefrontal, frontal, cingulate, insula [for GM only], parietal, temporal [divided into auditory, visual, and limbic regions], and occipital) and hemispheres (left and right) as repeated measures. The GM and WM data were analyzed in two stages-with and without correcting for intracranial volume (ICV) (i.e., values divided by ICV).

\section{Results}

\section{Influenza Infection}

All 12 gravid dams exposed intranasally to influenza showed evidence of infection (Figures 2A-2C). Viral shedding in nasal secretions was assessed for 2 weeks, but the self-limiting infection resolved on average by Day 7. Systemic activation was indicated by increased neopterin in many but not all females on Days 3 and 7. Significant increases in virusspecific $\operatorname{IgM}$ and $\operatorname{IgG}$ were seen in the mothers at 2 weeks, and elevated antibody was still present at birth. Newborn infants also had virus-specific antibody in their blood, although only IgG-not the IgM class - indicating the antibody was maternal and transplacentally derived. This interpretation was supported by a high correlation between influenza-specific IgG levels in maternal and neonatal blood $(r=.90)$ (Figure 2C). The absence of IgM in the neonate supported the conclusion that virus had not reached the fetal compartment and elicited a primary response (data not shown).

\section{Infant Measures}

All 19 infants were born naturally, and maternal infections did not affect gestation lengths or birth weights (Table 1). When tested at 2 weeks of age, infants from the influenza condition were similar to control subjects on Temperament, Motor Maturity, and Motor Activity measures, with the exception that Virus-exposed males performed more poorly than Control males on the Orientation subscale $(p=.012)$. Test items comprising the latter subscale included attention (e.g., duration of looking, distractibility) and ability to orient to visual and auditory stimuli.

\section{Mother-Infant Behavior}

Mother-infant interactions from 1 to 4 months indicated that offspring from influenzaexposed pregnancies spent significantly less time in contact with their mothers $[F(1,17)=$ $5.05, p=.04]$ and were more likely to move off the mother and explore the cage at an earlier age (Table 1). This earlier autonomy was accompanied by some signs of arousal, including an increased likelihood of vocalizing.

\section{Hypothalamic-Pituitary-Adrenal Responses}

Analysis of basal and stress-induced cortisol indicated no significant group differences when tested at 1.5 years of age. However, cortisol responses reflected the expected reactions to experimental conditions (e.g., associated with arousal, diurnal decline, and Dex suppression). Changes in cortisol were significant across the week of sampling $[F(5,85)=$ $54.45, p<.0001]$. Relocation to an unfamiliar room elicited a significant elevation from basal levels $(p<.0001)$, and afternoon cortisol remained elevated 2 days later $(p<.0001)$. Compared with the Acclimation morning, Dex was effective in reducing adrenal secretion on the following morning $(p<.0001)$. However, there was not a significant effect of 
prenatal virus exposure on any of these measures (Table 1). Similar basal and stress levels were found, and a comparable moderate suppression of cortisol was seen after Dex administration.

\section{Neuroimaging}

Global Brain Measures-Animals from virus-exposed pregnancies tended to have a reduced ICV $(4.4 \%$ smaller; $p=.086)$ with significantly less total GM $(5.6 \% ; p=.028)$ and cortical GM $(6.2 \% ; p=.029$; Table 2$)$. After controlling for ICV, reductions in cortical GM remained significant $(p=.031)$. They also had less total WM (3.6\%), although this difference did not reach statistical significance $(p=.091)$. After controlling for ICV, there were significant reductions of cerebellar WM in offspring from virus-exposed pregnancies $(p=.038)$. Overall the amount of cerebro-spinal fluid was also lower in these monkeys, in keeping with their smaller brain size, whereas the lateral ventricles revealed the opposite trend toward enlargement but clearly evident only in a subset of virus-exposed animals ( $p=$. 28, after controlling for ICV).

Cortical Volumes-Analyses of regional cortical volumes indicated that, before ICV correction, monkeys from virus-exposed pregnancies had less GM in prefrontal (bilateral), frontal (right), cingulate (bilateral), insula (right), parietal (bilateral), and temporal-auditory regions (bilateral). After correcting for the smaller total brain size, significant differences remained in the cingulate and parietal areas, whereas decreases in other regions were not greater than the general reduction in overall brain size (Figure 3, Table 3). Significant differences in WM were restricted primarily to the parietal lobes and the left temporalauditory region before ICV corrections. The WM volume in the left parietal region remained significantly smaller after accounting for ICV. Interestingly, after correcting for ICV, cingulate WM was proportionally greater bilaterally in animals from virus-exposed pregnancies.

Subcortical Regions-Virus-exposed offspring had significantly smaller amygdalae ( $p$ $=.04$ ) than control subjects in both the right and left hemispheres but in analyses of uncorrected data only. No group differences were observed for the hippocampus and striatum (Table S2 in Supplement 1).

\section{Associations Between Influenza Severity and Brain Alterations}

Brain regions that were still significantly different after ICV correction were analyzed with respect to the magnitude of the mothers' antibody responses. Significant negative correlations were found for the cingulate (left $r=-.64, p=.027$; right $r=-.73, p=.007$ ). Because enlarged lateral ventricles are characteristic of the structural neuropathology observed with schizophrenia, we also assessed the relationship between ventricular volume and $\mathrm{IgG}$. Correlational analyses for the 12 virus-exposed animals indicated that the size of their lateral ventricles was associated with the mothers' antibody response (left $r=.67, p=$. 017 ; right $r=.75, p=.005)$. Enlargement of the lateral ventricles was similarly correlated with influenza IgG in neonatal blood. However, the association with amount of viral shedding on Day 3 was only a statistical trend $(r=.53, p=.077)$, and the maternal neopterin response on Day 3 was not predictive.

\section{Discussion}

Our results indicate that, even in the absence of an effect on gestation length and birth weight, prenatal infection can significantly reduce overall brain size, especially the amount of GM in the young monkey. These alterations are likely to persist, given that the reduced volumes were evident at a stage equivalent to late-childhood in humans, when the monkey 
brain is nearly $80 \%$ of adult size (38). It is possible that the observed structural differences and loss of brain volume would manifest later in development as behavioral impairments. Common diathesis models proposed in humans highlight an emergent vulnerability for psychopathology during adolescence, following the onset of puberty $(39,40)$. In many prenatal infection studies with rats, behavioral abnormalities are also reported to first emerge after puberty in adulthood $(6,11,17)$. The peripubertal period could therefore provide a window of opportunity for pharmacological interventions (41). Our findings support both epidemiological research in humans $(8,42,43)$ and studies on prenatal infection in rodents $(13,15)$, although ours is the first to investigate influenza during pregnancy in a primate species. The larger brain size of this species enables the use of in vivo neuroimaging to evaluate the monkey's brain development, which has important translational relevance.

The nature and extent of the brain volume reductions seen in the monkey bore most in common with the structural abnormalities frequently found in schizophrenia. This includes reduced total brain and cortical GM volumes, ventricular enlargement, and regional decreases in frontal cortex and temporal-limbic structures (44-51). These structural differences have been reported to be present at first episode and have been recently shown in longitudinal MRI studies to precede the onset of clinical symptoms $(52,53)$. Support that the neural differences are antecedent is also evident in studies on adolescents and young adults at genetic risk for schizophrenia, who have smaller prefrontal lobe volumes (54) and reduced GM in right frontal, cingulate, temporal, and right parietal lobes (55). Mothers with schizophrenia were also found to gestate fetuses with larger lateral ventricles, providing the earliest etiologic evidence that brain development might already be abnormal during the fetal period (56).

Offspring from the influenza condition evinced a reduction in total brain volume (4.4\%); therefore, all regional analyses were also analyzed with respect to ICV, which revealed whether regional volumes were differentially affected. After ICV-correction, the extent of the reduction in regional GM was less pronounced, but volume decreases were still evident in the frontal (right, approximately 3\%) and parietal lobes (approximately 5\%) and the cingulate gyrus (approximately 7\%). Given the late gestational timing of the infection, it is understandable that reductions were less pronounced in the occipital lobe, which matures early in the fetal monkey (57).

The effects on WM were less pervasive than on GM. Regional analyses revealed the most significant volume loss occurred in the parietal lobe, both before and after ICV-correction (approximately $8 \%$ and approximately $5 \%$, respectively). In contrast, cingulate WM was significantly increased in virus-exposed animals, both before and after ICV-correction (approximately $6 \%$ and approximately $13 \%$, respectively). The increase in cingulate WM was of equal magnitude to the reduction of GM in this region. Few studies have systematically examined WM in the cingulate of individuals with schizophrenia. However, two recent articles reported a decrease in GM accompanied by increased WM in the cingulate $(58,59)$. It might also be that more sophisticated MR approaches, such as diffusion tension imaging, are required to detect other subtle impairments in WM integrity (60-62).

The $8 \%$ enlargement of the monkeys' lateral ventricles was not statistically significant, but considerable individual variation was evident in the influenza-exposed group. Differences in the maternal response to infection likely mediated the differential impact on ventricular volume given that there was a significant correlation between ventricular size and amount of antibody present in both the mother and newborn infant. Viral shedding and elevated neopterin persisted for $<1$ week, although antibody titers were still increased at term, 6 weeks after infection. Virus-specific maternal $\operatorname{IgG}$ was also actively transferred into the fetal compartment, in keeping with the high placental transfer of maternal IgG in primates (28). 
Despite the large brain effects, early behavioral functioning was similar to control subjects and, there were no overt differences in stress reactivity. One of the few behavioral differences seen in the flu-exposed offspring was a more rapid autonomy from the mother by 4 months of age, an effect that has also been observed in monkeys generated from psychologically stressed or iron-deficient pregnancies (63). The reason for this precocious behavior is not known, but it supports our conclusion that physical growth and general maturation were not slowed by the viral infection. Further examination of their emotional reactivity under more arousing conditions and in response to drug challenges as they mature through the pubertal transition might reveal that the reduced cortical volume had more consequential behavioral effects.

Species differences in the extent of behavioral findings might also reflect the virulence of the influenza strain employed: a more moderate infection induced by A/Sydney (H3N2) versus the neurovirulent $\mathrm{A} / \mathrm{NWS} / 33$ (H1N1) used in most recent rodent projects. This point is important to consider when interpreting findings from human studies assessing the risks posed by different strains, such as after the 1957 or current H1N1 pandemic $(5,10,42,64)$. In rodent models, the extent of the behavioral impact is also usually associated with the magnitude of the pathogenic challenge, with larger effects provoked by administration of endotoxin to the pregnant female than after viral infections (65).

In keeping with the normalcy of the monkeys' physical appearance and behavior, their hypothalamic-pituitary-adrenocortical responses to stress and to Dex were not perturbed. This finding of normal cortisol activity differs from rodent findings of greater stress reactivity after prenatal infection and altered hypothalamic-pituitary-adrenocortical regulation after endotoxin administration $(66,67)$. The absence of a difference in cortisol also supports our view that the brain effects were specific to the viral infection. In contrast, prenatal stress manipulations can elevate the infant monkey's emotional reactivity and adrenal responses to Dex and decrease the size of its hippocampus (68). To rule out nonspecific effects due to maternal disturbance during the infection phase, our control animals received the same handling procedures except for the flu exposure (i.e., sham infections).

Because influenza infections vary so much across strains and species, it is important to consider the degree of immune activation and potential lethality (69). Rodent models provide the most compelling evidence for the role of maternal cytokines in mediating the effects on neurodevelopment. However, because mice are not a natural host for influenza (70), brain-adapted, neurovirulent strains are often used, which sometimes results in fetal or maternal loss $(13,14)$. In contrast, when infecting monkeys, it was possible to use a lessvirulent strain derived from human patients $(71,72)$ and to assess a moderate, self-limiting infection that more closely mirrors human illness.

Although the precise mechanisms derailing brain development have yet to be identified, it is clear that the fetus need not be directly exposed to the virus. Instead, secondary mediators generated by the maternal immune response have been implicated. Proinflammatory cytokines, such as interleukin- 6 , can affect placental functioning, transfer to the amniotic fluid, and enter fetal circulation (73). Placentally generated cytokines can in turn provoke cytokine synthesis by the fetus $(4,74)$, and these soluble substances and circulating leukocytes readily permeate the immature blood-brain barrier and central nervous system $(75,76)$.

In summary, the monkey offers a valuable model with translational relevance for assessing the impact of prenatal infection on brain development. The large effects on the brain, in the absence of more pronounced behavioral or hormone differences, suggests that it will be 
important to assess the affected animals under challenging conditions or after the second period of neuronal pruning and cortical thinning at puberty, when psychological disturbances become more manifest in adolescent humans. Future research also needs to delineate the impact of viral exposure at earlier points in pregnancy and to assess the benefits of prior maternal immunization against influenza to lessen the neural sequelae of infection.

\section{Supplementary Material}

Refer to Web version on PubMed Central for supplementary material.

\section{Acknowledgments}

This research was supported by funding from the National Institute of Allergy and Infectious Diseases (AI067518). The author SJS had a National Research Service Awards fellowship from the National Institute of Mental Health (F31 MH076606), and CC and GL receive partial salary support from a National Institute of Child Health and Human Development grant (HD383386). Additional support was provided by the University of North Carolina Conte Center for Schizophrenia Research [MH 064065] and the University of North Carolina Neurodevelopmental Disorders Research Center (HD 03110).

Special acknowledgements are due for M. Luck, H. Crispen, and M. Schneider for technical assistance with the animal care and experimental procedures.

\section{References}

1. Brown AS. Prenatal infection as a risk factor for schizophrenia. Schizophr Bull. 2006; 32:200-202. [PubMed: 16469941]

2. Dammann O, Leviton A. Maternal intrauterine infection, cytokines and brain damage in the preterm newborn. Pediatr Res. 1997; 42:1-8. [PubMed: 9212029]

3. Gilmore JH, Jarskog LF, Vadlamudi S, Lauder JM. Prenatal infection and risk for schizophrenia: IL-1, IL-6, and TNF inhibit cortical neuron dendrite development. Neuropsychopharmacology. 2004; 29:1221-1229. [PubMed: 15085088]

4. Gilmore JH, Jarskog LF, Vadlamudi S. Maternal Poly I: C exposure during pregnancy regulates $\mathrm{TNF} \alpha, \mathrm{BDNF}$, and NGF expression in neonatal brain and the maternal-fetal unit of the rat. $\mathrm{J}$ Neuroimmunol. 2005; 159:106-112. [PubMed: 15652408]

5. Mednick SA, Machon RA, Huttunen MO, Bonett D. Adult schizophrenia following prenatal exposure to an influenza Epidemic. Arch Gen Psychiatry. 1988; 45:189-192. [PubMed: 3337616]

6. Meyer U, Feldon J, Schedlowski M, Yee BK. Towards an immuno-precipitated neurodevelopmental animal model of schizophrenia. Neurosci Biobehav Rev. 2005; 29:913-947. [PubMed: 15964075]

7. Irving WL, James DK, Stepheson T, Laing P, Jameson C, Oxford JS, et al. Influenza virus infection in the second and third trimesters of pregnancy: A clinical and seroepidemiological study. $\mathrm{Br} \mathrm{J}$ Obstet Gynaecol. 2000; 107:1282-1289.

8. Brown AS, Begg MD, Gravenstein S, Schaefer CA, Wyatt RJ, Bresnahan M, et al. Serologic evidence of prenatal influenza in the etiology of schizophrenia. Arch Gen Psychiatry. 2004; 61:774780. [PubMed: 15289276]

9. Ellman LM, Yoken RH, Buka SL, Torrey F, Cannon TD. Cognitive functioning prior to the onset of psychosis: The role of fetal exposure to serological determined infection. Biol Psychiatry. 2009; 65:1040-1047. [PubMed: 19195645]

10. McGrath JJ, Pemberton MR, Welham JL, Murray RM. Schizophrenia and the influenza epidemics of 1954, 1957, and 1959: A southern hemisphere study. Schizophr Res. 1994; 14:1-8. [PubMed: 7893616]

11. Nawa H, Takei N. Recent progress in animal modeling of immune inflammatory processes in schizophrenia: Implication of specific cytokines. Neurosci Res. 2006; 56:2-13. [PubMed: 16837094] 
12. Fatemi SH, Emamian ES, Akher P, Sedgewick J, Thuras P, Bailey K, et al. Human influenza viral infection in utero increases nNOS expression in hippocampi of neonatal mice. Synapse. 1998; 29:84-88. [PubMed: 9552178]

13. Fatemi SH, Emamian ES, Kist D, Sidwell RW, Nakajima K, Akhter P, et al. Defective corticogenesis and reduction in reelin immunoreactivity in cortex and hippocampus of prenatally infected neonatal mice. Mol Psychiatry. 1999; 4:145-154. [PubMed: 10208446]

14. Fatemi SH, Emamian ES, Sidwell RW, Kist DA, Stary JM, Earle JA, et al. Human influenza viral infection in utero alters glial fibrillary acidic protein immunoreactivity in the developing brains of neonatal mice. Mol Psychiatry. 2002; 7:633-640. [PubMed: 12140787]

15. Fatemi SH, Reutiman TJ, Folsom TD, Huang H, Oishi K, Mori S, et al. Maternal infection leads to abnormal gene regulation and brain atrophy in mouse offspring: Implications for genesis of neurodevelop-mental disorders. Schizophr Res. 2008; 99:56-70. [PubMed: 18248790]

16. Winter C, Djodari-Irani A, Sohr R, Morgenstern R, Feldon J, Juckel G, Meyer U. Prenatal immune activation leads to multiple changes in basal neurotransmitter levels in the adult brain: Implications for brain disorders of neurodevelopmental origin such as schizophrenia. Int J Neuropsychopharmacol. 2009; 12:513-524. [PubMed: 18752727]

17. Shi L, Fatemi SH, Sidwell RW, Patterson PH. Maternal influenza infection causes marked behavioral and pharamacological changes in the offspring. J Neurosci. 2003; 23:297-302. [PubMed: 12514227]

18. Clancy B, Finlay BL, Darlington RB, Anand KJS. Extrapolating brain development from experimental species to humans. Neurotoxicology. 2007; 28:931-937. [PubMed: 17368774]

19. Clancy B, Darlington B, Finlay BL. Translating developmental time across mammalian species. Neuroscience. 2001; 105:7-17. [PubMed: 11483296]

20. LaMantia AS, Rakic P. Axon overproduction and elimination in the corpus callosum of the developing rhesus monkey. J Neurosci. 1990; 10:2156-2175. [PubMed: 2376772]

21. Collie MH, Sweet C, Cavanagh D, Smith H. Association of foetal wastage with influenza infection during ferret pregnancy. Br J Exp Pathol. 1978; 59:190-195. [PubMed: 656319]

22. Wesley RD. Exposure or Sero-positive gilts to swine influenza virus may cause a few stillbirths per litter. Can J Vet Res. 2004; 68:215-217. [PubMed: 15352547]

23. Harris JW. Influenza occurring in pregnant women: A statistical study of thirteen hundred and fifty cases. JAMA. 1919; 72:978-980.

24. Aronsson F, Lannebo C, Paucar M, Brask J, Kristensson K, Karlsson H. Persistence of viral RNA in the brain of offspring to mice infected with influenza A/WSN/33 virus during pregnancy. J Neurovirol. 2002; 8:353-357. [PubMed: 12161820]

25. Gu J, Xie Z, Gao Z, Liu J, Korteweg C, Ye J, et al. H5N1 infection of the respiratory tract and beyond: A molecular pathology study. Lancet. 2007; 370:1137-1145. [PubMed: 17905166]

26. Battaglia FC. Placental transport: A function of permeability and perfusion. Am J Clin Nutr. 2007; 85:591S-5597. [PubMed: 17284761]

27. Coe CL, Kemnitz JW, Schneider ML. Vulnerability of placental antibody transfer and fetal complement synthesis to disturbance in the pregnant monkey. J Med Primatol. 1993; 22:294-300. [PubMed: 8289218]

28. Coe CL, Lubach GR, Izard KM. Progressive improvement in the transfer of maternal antibody across the order primates. Am J Primatol. 1994; 32:51-55.

29. Moffett A, Loke C. Immunology of placentation in eutherian mammals. Nat Rev Immunol. 2006; 6:584-594. [PubMed: 16868549]

30. Pere MC. Materno-foetal exchanges and utilisation of nutrients by the foetus: Comparison between species. Reprod Nutr Dev. 2003; 43:1-15. [PubMed: 12785446]

31. Rakic P. Specification of cerebral cortical areas. Science. 1988; 241:170-176. [PubMed: 3291116]

32. Portman OW, Neuringer M, Alexander M. Effects of maternal and long-term postnatal protein malnutrition on brain size and composition in rhesus monkey. J Nutr. 1987; 11:1844-1851. [PubMed: 3681475]

33. Rigo JJR, Szelenyi J, Selmeczy Z, Papp Z, Vizi ES. Endotoxin-induced TNF production changes inversely to its plasma level during pregnancy. Eur J Obstet Gynecol. 2004; 114:236-238. 
34. Schneider ML, Suomi SJ. Neurobehavioral assessment in rhesus monkey neonates (Macaca mulatta): Developmental changes, behavioral stability, and early experience. Infant Behav Dev. 1992; 15:155-177.

35. Schneider ML, Roughton EC, Lubach GR. Moderate alcohol consumption and psychological stress during pregnancy induces attention and neuromotor impairments in primate infants. Child Dev. 1997; 68:747-759.

36. Rosenblum, LA. The ontogeny of mother-infant relations in macaques. In: Moltz, H., editor. The Ontogeny of Vertebrate Behavior. Academic Press; New York: 1971. p. 315-367.

37. Styner M, Knickmeyer RC, Joshi S, Coe CL, Short SJ, Gilmore JH. Automatic brain segmentation in rhesus monkeys. Spie PP. 2007; 65122:L65121-L65128.

38. Knickmeyer RC, Styner M, Short SJ, Lubach GR, Kang C, Hamer R, et al. Maturational trajectories of cortical brain development through the pubertal transition: Unique species and gender differences in the monkey revealed through structural magnetic resonance imaging. Cereb Cortex. 2009 published online ahead of print August 24.

39. Rapoport JL, Addington AM, Frangou S, Psych MR. The neurodevelopmental model of schizophrenia: Update 2005. Mol Psychiatry. 2005; 10:434-449. [PubMed: 15700048]

40. Spear, LP. Neurodevelopment during adolescence. In: Cicchetti, D.; Walker, EF., editors. Neurodevelopmental Mechanisms in Psychopathology. Cambridge University Press; Cambridge: 2003. p. 62-83.

41. Piontkewitz Y, Assaf Y, Weiner I. Clozapine administration in adolescence prevents postpubertal emergence of brain structural pathology in an animal model of schizophrenia. Biol Psychiatry. 2009; 66:1038-1046. [PubMed: 19726031]

42. Brown AS, Susser ES. In utero infection and adult schizophrenia. Ment Retard Dev Disabil Res Rev. 2002; 8:51-57. [PubMed: 11921387]

43. Buka SL, Tsuang MT, Torrey EF, Klebanoff MA, Berstein D, Yolken RH. Maternal infections and subsequent psychosis among offspring. Arch Gen Psychiatry. 2001; 58:1032-1037. [PubMed: 11695949]

44. Cannon TD, Thompson PM, van Erp TGM, Toga AW, Veli-Pekka P, Huttunen M, et al. Cortex mapping reveals regionally specific patterns of genetic and disease-specific gray-matter deficits in twins discordant for schizophrenia. Proc Natl Acad Sci U S A. 2002; 99:3228-3233. [PubMed: 11867725]

45. Pantelis C. Structural brain imaging evidence for multiple pathological processes at different stages of brain development in schizophrenia. Schizophr Bull. 2005; 31:672-696. [PubMed: 16020551]

46. Wright I, Rabe-Hesketh S, Woodruff P, David A, Murray R, Bullmore E. Meta-analysis of regional brain volumes in schizophrenia. Am J Psychiatry. 2000; 157:16-25. [PubMed: 10618008]

47. Akbarian S, Viñuela A, Kim JJ, Potkin SG, Bunney WEJ, Jones EG. Distorted distribution of nicotinamide-adenine dinucleotide phosphate-diaphorase neurons in temporal lobe of schizophrenics implies anomalous cortical development. Arch Gen Psychiatry. 1993; 50:178-187. [PubMed: 7679892]

48. Arnold SE, Trojanowski JQ. Recent advances in defining the neuropathology of schizophrenia. Acta Neuropathol. 1996; 92:217-231. [PubMed: 8870823]

49. Andreasen NC, Flashman L, Flaum M, Arndt S, Swayze V, O'Leary DS, et al. Regional brain abnormalities in schizophrenia measured with magnetic resonance imaging. JAMA. 1994; 272:1763-1769. [PubMed: 7966925]

50. Harrison PJ, Weinberger DR. Schizophrenia genes, gene expression, and neuropathology: On the matter of their convergence. Mol Psychiatry. 2004; 10:40-68. [PubMed: 15263907]

51. Honea R, Crow TJ, Passingham D, Mackay CE. Regional deficits in brain volume in schizophrenia: A meta-analysis of voxel-based morphometry studies. Am J Psychiatry. 2005; 162:2233-2245. [PubMed: 16330585]

52. Steen RG, Mull C, McClure R, Hamer RM, Liberman JA. Brain volume in first-episode schizophrenia: Systematic review and meta-analysis of magnetic resonance imaging studies. $\mathrm{Br} \mathrm{J}$ Psychiatry. 2006; 188:510-518. [PubMed: 16738340] 
53. Cahn W, Hulshoof HE, Bongers M, Schnack HG, Mandl RCW, Van Haren NEM, et al. Brain morphology in antipsychotic-naïve schizophrenia: A study of multiple brain structures. Br J Psychiatry. 2002; 181:266-272.

54. Lawrie SM, Whalley HC, Abukmeil SS, Kestelman JN, Donnelly L, Miller P, et al. Brain structure, genetic liability, and psychotic symptoms in subjects at high risk of developing schizophrenia. Biol Psychiatry. 2001; 49:811-823. [PubMed: 11343678]

55. Job DE, Whalley HC, Johnstone EC, Lawrie SM. Grey matter changes over time in high risk subjects developing schizophrenia. Neuroimage. 2005; 25:1023-1030. [PubMed: 15850721]

56. Clarke MC, Cannon M, Hogg MW, Marks MN, Conroy S, Pawlby SJ, et al. Foetal brain development in offspring of women with psychosis. Br J Psychiatry. 2007; 190:445-446. [PubMed: 17470961]

57. Rakic P. Radial versus tangential migration of neuronal clones in the developing cerebral cortex. Proc Natl Acad Sci U S A. 1995; 92:11323-11327. [PubMed: 8524778]

58. Hazlett E, Buchsbaum M, Haznedar M, Newmark R, Goldstein K, Zelmanova Y, et al. Cortical gray and white matter volume in unmedicated schizotypal and schizophrenia patients. Schizophr Res. 2008; 101:111-123. [PubMed: 18272348]

59. van Haren NEM, Hulshoff Pol HE, Schnack HG, Cahn W, Brans R, Carati I, et al. Progressive brain volume loss in schizophrenia over the course of the illness: Evidence of maturational abnormalities in early adulthood. Biol Psychiatry. 2007; 63:106-113. [PubMed: 17599810]

60. Lim KO, Helpern JA. Neuropsychiatric applications of DTI-A review. NMR Biomed. 2002; 15:587-593. [PubMed: 12489105]

61. Ardekani BA, Nierenberg J, Hoptman MJ, Javitt DC, Lim KO. MRI study of white matter diffusion anisotropy in schizophrenia. Neurore-port. 2003; 14:2025-2029.

62. Kubicki M, McCarley R, Westin CF, Park HJ, Maier S, Kikinis R, et al. A review of diffusion tensor imaging studies in schizophrenia. J Psychiatr Res. 2007; 41:15-30. [PubMed: 16023676]

63. Coe CL, Lubach GR, Shirtcliff E. Maternal stress during pregnancy predisposes for iron deficiency in infant monkeys impacting innate immunity. Pediatr Res. 2007; 61:520-524. [PubMed: 17413860]

64. Jamieson DJ, Honein MA, Rasmusen SA, Willaims JL, Swerdlow DL, Biggerstaff MS, et al. H1N1 2009 influenza virus infection during pregnancy in the USA. Lancet. 2009; 374:451-458. [PubMed: 19643469]

65. Fortier ME, Luheshi GN, Boksa P. Effects of prenatal infection on PPI in rat depend upon nature of the infectious agent and stage of pregnancy. Behav Brain Res. 2007; 181:270-277. [PubMed: 17553574]

66. Hodyl NA, Krivanek KM, Lawrence E, Clifton VL, Hodgson DM. Prenatal exposure to a proinflammatory stimulus causes delays in the development of the innate immune response to LPS in the offspring. J Neuroimmunol. 2007; 190:61-71. [PubMed: 17804085]

67. Reul JMHM, Stec I, Wiegers GJ, Labeur MS, Linthorst ACE, Arzt E, et al. Prenatal immune challenge alters the hypothalamic-pituitary-adrenocortical axis in adult rats. J Clin Invest. 1994; 93:2600-2607. [PubMed: 8200998]

68. Coe CL, Kramer M, Czeh B, Gould E, Reeves AJ, Kirschbaum C, Fuchs E. Prenatal stress diminishes neurogenesis in the dentate gyrus of juvenile rhesus monkeys. Biol Psychiatry. 2003; 54:1025-1034. [PubMed: 14625144]

69. Subbarao, K.; Swayne, D.; Olsen, CW. Epidemiology and control of human and animal influenza. In: Kawaoka, Y., editor. Influenza Virology: Current Topics. Caister Academic Press; Wymondham, United Kingdom: 2006. p. 229-280.

70. Sidwell, RW. The mouse model of influenza virus infection. In: Zak, O.; Sande, MA., editors. Handbook of Animal Models of Infection: Experimental Models in Antimicrobial Therapy. Academic Press; London, United Kingdom: 1999. p. 981-988.

71. Rimmelzwaan GF, Kuiken T, van Amerongen G, Bestebroer TM, Fouchier TA, Osterhous AD. Pathogenesis of influenza A (H5N1) virus infection in a primate model. J Virol. 2001; 75:66876691. [PubMed: 11413336] 
72. Rimmelzwaan GF, Kuiken T, van Amerongen G, Bestebroer TM, Fouchier TA, Osterhous AD. A primate model to study the pathogenesis of influenza A (H5N1) virus infection. Avian Dis. 2003; 47:931-933. [PubMed: 14575089]

73. Smith S, Li J, Garbett K, Mirnics K, Patterson P. Maternal immune activation alters fetal brain development through interleukin-6. J Neurosci. 2007; 27:10695-10702. [PubMed: 17913903]

74. Urakubo A, Jarskog LF, Lieberman JA, Gilmore JH. Prenatal exposure to maternal infection alters cytokine expression in the placenta, amniotic fluid, and fetal brain. Schizophr Res. 2001; 47:2736. [PubMed: 11163542]

75. Meyer U, Nyffeler M, Engler A, Urwyler A, Schedlowski M, Knuesel I, et al. The time of prenatal immune challenge determines the specificity of inflammation-mediated brain and behavioral pathology. J Neurosci. 2006; 26:4752-4762. [PubMed: 16672647]

76. Rees S, Harding R. Brain development during fetal life: Influences of the intra-uterine environment. Neurosci Lett. 2004; 361:111-114. [PubMed: 15135906] 


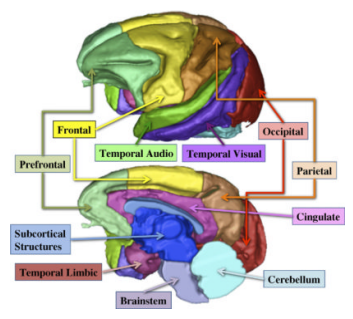

Figure 1.

Illustration of the parcellation of cortical areas used for regional analysis of gray matter and white matter volumes in infant monkeys from control and influenza virus-exposed pregnancies. 


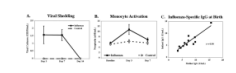

Figure 2.

Local and systemic measures confirming infection with influenza virus. (A) Viral shedding confirmed by nasal swabs from flu-exposed mothers. (B) Systemic reaction evident by increased neopterin levels at 3-7 days after exposure to influenza. (C) Significant correlation between the levels of flu-specific antibody (immunoglobulin $\mathrm{G}[\mathrm{IgG}]$ class) found in virus-exposed mothers and their neonates at 2-3 days after birth. 




Figure 3.

To illustrate the magnitude of the cortical effects of prenatal infection, Figure 3 portrays how much smaller the gray matter volumes were in monkeys from flu-exposed pregnancies relative to controls. Regional reductions ranging from 4-7\% reached statistical significance $(* p<.05)$, with more modest decrements evident in the posterior cortex, including occipital lobe and the temporal-limbic area. 
Table 1

Minimal Difference in Physical Growth, Neonatal Maturation, and Adrenal Activity in Control and Influenza Virus-Exposed Infants

\begin{tabular}{|c|c|c|c|}
\hline Measure & Control Mean $\pm \mathrm{SE}$ & Influenza Mean $\pm \mathrm{SE}$ & $p$ \\
\hline Gestation Length (days) & $171 \pm 1$ & $169 \pm 2$ & .48 \\
\hline Birth Weight (g) & $496 \pm 37$ & $529 \pm 16$ & .46 \\
\hline \multicolumn{4}{|l|}{ NAS (0-2) } \\
\hline Temperament & $1.06 \pm .08$ & $1.01 \pm .06$ & .36 \\
\hline Orient & $1.26 \pm .15$ & $1.11 \pm .11$ & $.27^{a}$ \\
\hline Motor activity & $1.03 \pm .13$ & $.93 \pm .10$ & .28 \\
\hline Motor activity & $1.12 \pm .12$ & $.98 \pm .09$ & .45 \\
\hline \multicolumn{4}{|c|}{ Infant Independence ( $\%$ of time) } \\
\hline Month 1 & $4 \pm 3$ & $8 \pm 2$ & .31 \\
\hline Month 2 & $16 \pm 5$ & $26 \pm 4$ & .14 \\
\hline Month 3 & $25 \pm 5$ & $41 \pm 4$ & $.03^{a}$ \\
\hline \multicolumn{4}{|l|}{ Cortisol Levels ( $\mu \mathrm{g} / \mathrm{dL}$ ) } \\
\hline Basal & $35 \pm 5$ & $29 \pm 2$ & .14 \\
\hline Stress & $78 \pm 3$ & $69 \pm 5$ & .28 \\
\hline Acclimation AM & $57 \pm 5$ & $58 \pm 4$ & .73 \\
\hline Acclimation PM & $46 \pm 3$ & $43 \pm 2$ & .25 \\
\hline DST AM & $38 \pm 8$ & $35 \pm 6$ & .76 \\
\hline DST PM & $29 \pm 5$ & $23 \pm 4$ & .51 \\
\hline
\end{tabular}

Analysis of variance of the neurobehavioral assessment scale (NAS) showed a condition $\times$ gender interaction $(p=.003)$ on the Orientation subscale. Follow-up tests showed that virus-exposed males scored lower than control males on this measure $(p=.012)$. Control $n=7$, Influenza $n=$ 12 .

DST, Dexamethasone Suppression Test.

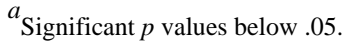




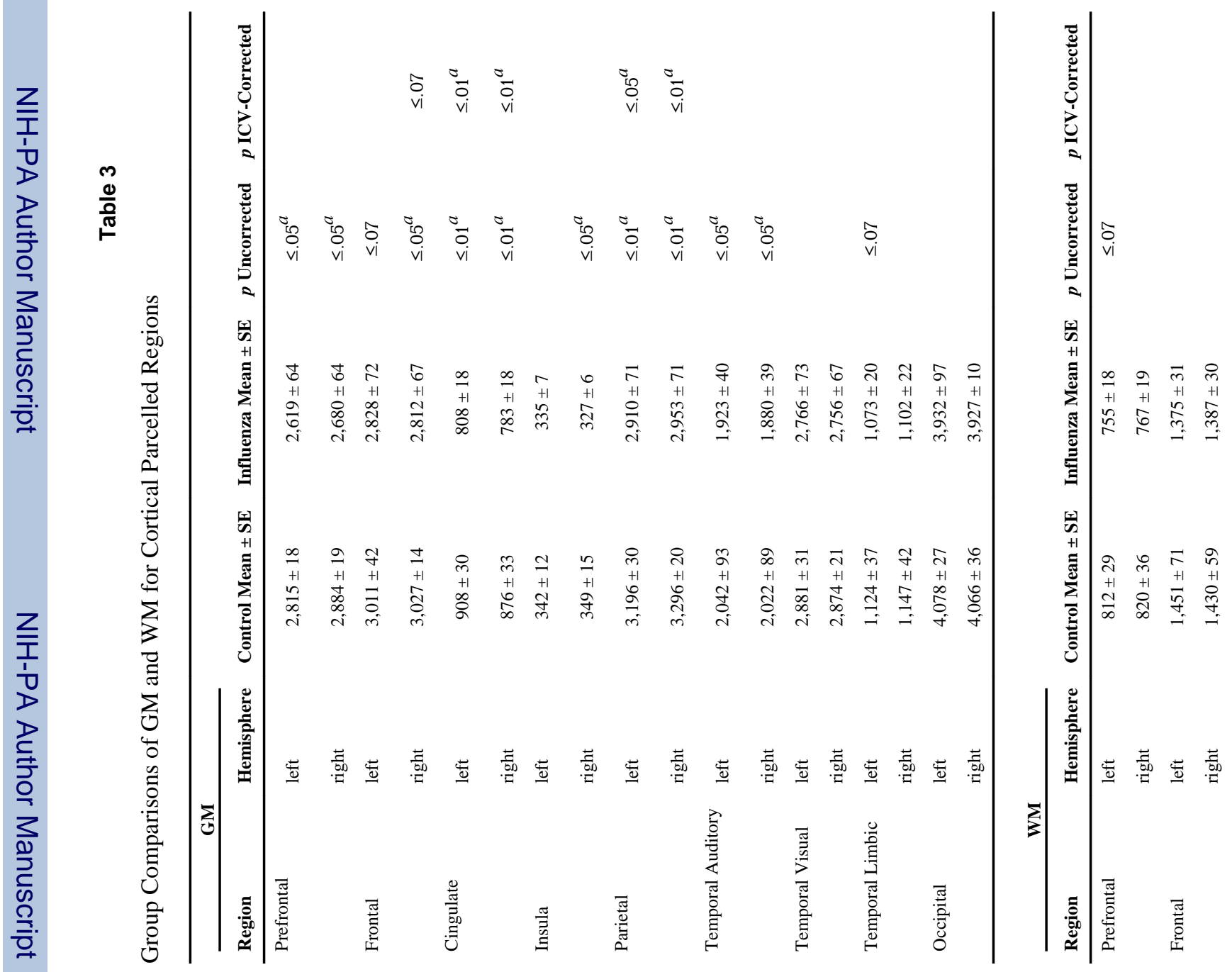
Biol Psychiatry. Author manuscript; available in PMC 2011 December 11. 


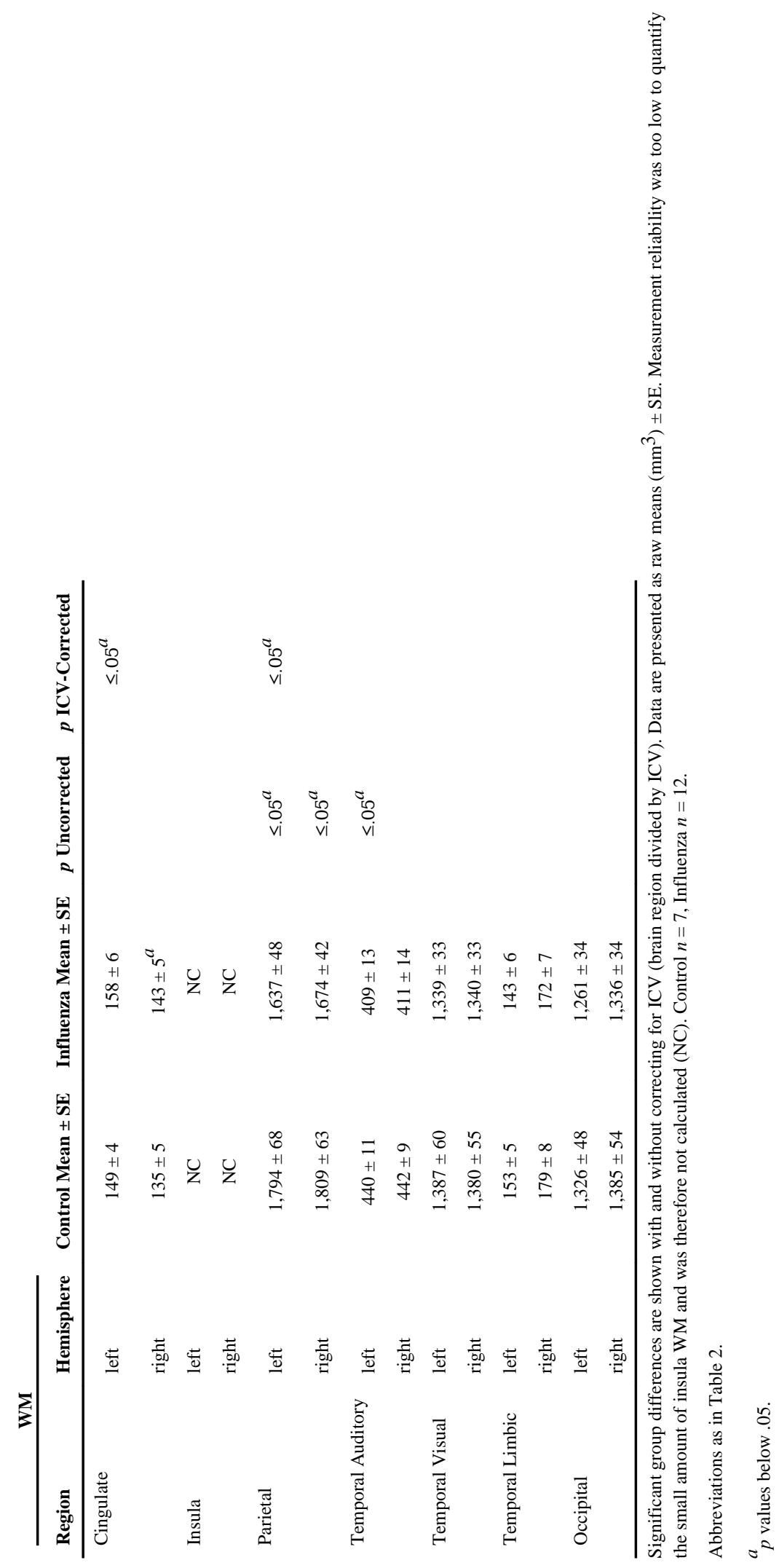

